\title{
Quercetin-loaded mixed micelles exhibit enhanced cytotoxic efficacy in non-small cell lung cancer in vitro
}

\author{
MING-HONG ZHAO ${ }^{1 *}$, LIN YUAN ${ }^{1 *}$, LING-YUN MENG $^{1}$, JIAN-LING QIU ${ }^{1}$ and CHUN-BIN WANG ${ }^{2}$ \\ ${ }^{1}$ Department of Oncology, Jianhu People's Hospital, Yancheng, Jiangsu 224700; \\ ${ }^{2}$ Department of Oncology, Yancheng Third People's Hospital, Yancheng, Jiangsu 224001, P.R. China
}

Received May 19, 2016; Accepted April 7, 2017

DOI: $10.3892 /$ etm.2017.5230

\begin{abstract}
In the present study, quercetin (QUR)-loaded mixed micelles (QUR-M) were prepared with the aim of improving the physicochemical and anticancer efficacy of QUR in lung cancer cells. The mixed micelles comprised tocopheryl polyethylene glycol 1000 succinate (TPGS) and a 1,2-distearoyl-sn-glycero-3-phosphatidylethanolamine derivative of polyethylene glycol. The nanosized QUR-M exhibited a $\mathrm{pH}$-responsive and controlled release of QUR that is likely to be beneficial in cancer treatment. The results of an MTT assay clearly demonstrated that the anticancer effect of QUR-M in A549 cancer cells was stronger compared with that of free QUR at 24 and $48 \mathrm{~h}$ time points. The half-maximal inhibitory concentrations of QUR and QUR-M were observed to be 12.45 and $6.42 \mu \mathrm{g} / \mathrm{ml}$, respectively. When stained with Hoechst 33342 and observed using a confocal laser scanning microscope, A549 cells treated with QUR-M exhibited severe chromatin condensation and apoptotic body formation of the nuclei. Overall, high intracellular uptake, sustained drug release and the presence of TPGS in the mixed micelles may result in an increased inhibitory effect against cell proliferation and improved therapeutic efficacy in lung cancers.
\end{abstract}

\section{Introduction}

Lung cancer is one of the prevalent causes of cancer-related mortality across the world in males and females $(1,2)$. In the United States alone, lung cancer accounts for $>30 \%$ of cancer-related mortalities (3). The total number of mortalities due to lung cancer exceeds the mortality rate of colon, prostate and breast cancers combined with a poor 5-year survival rate

Correspondence to: Dr Chun-Bin Wang, Department of Oncology, Yancheng Third People's Hospital, 75 Juchang Road, Yancheng, Jiangsu 224001, P.R. China

E-mail: wangchunbin1968@126.com

${ }^{*}$ Contributed equally

Key words: quercetin, lung cancer, mixed micelles, apoptosis, $\mathrm{pH}$-responsive of $\sim 15 \%$ (4). Among lung cancers, non-small cell lung cancer (NSCLC) constitutes $80 \%$ of cases (5). The present treatment options for lung cancer include chemotherapy, radiation and surgery. Chemotherapy is one of the prominent options for the effective treatment of lung cancers. However, cancer treatment with routine chemotherapeutic agents results in severe drug-related systemic side effects, which limits the potential clinical benefits (6-8).

In this regard, natural components from plant resources offer numerous options for the effective replacement of routine chemotherapeutic agents. Quercetin (QUR; $3,5,7,3$ ',4'-pentahydroxyflavone) is a naturally occurring flavonoid that is widely present in fruits, vegetables and leaves $(9,10)$. QUR is considered an excellent antioxidant owing to its high number of hydroxyl groups (11). The numerous applications of QUR include the treatment of allergy, inflammation, arteriosclerosis and bleeding (12). QUR has also received attention as a chemoprotective agent exhibiting a potent antiproliferative effect on cancer cells without any effect on normal cells $(13,14)$. QUR has been indicated to induce the apoptosis of cancer cells by blocking cell cycle progression at different phases of the cell cycle and modulating signaling pathways (15). QUR actively suppresses cancer-related processes such as apoptosis, proliferation and cancer metastasis (16). Despite its promising anticancer applications, the therapeutic activity of QUR is severely hindered owing to its poor aqueous solubility and high hydrophobicity. In addition, the natural form of QUR has been reported to possess a short half-life in the systemic circulation (17). Therefore, efforts are required to improve the physicochemical characteristics of QUR and thereby enhance its anticancer effects.

Nanoparticulate drug delivery systems have been developed to encapsulate hydrophobic small molecules, increase their solubility in body fluids and improve their anticancer efficacy. Biodegradable nanocarriers have been reported to improve the stability of encapsulated compounds and control their release in the systemic environment (18-20). In this context, self-assembled polymeric micelles are considered to be one of the most promising drug delivery systems for small molecules (21). The core-shell morphology of micelles protects the drug in the harsh systemic environment (22). The core material is used to load the drug while a shell composed of polyethylene glycol (PEG) provides excellent biocompatibility and protective effects and prolongs the blood circulation 
time of the drug (23). In the present study, mixed micelles consisting of a 1,2-distearoyl-sn-glycero-3-phosphatidylethanolamine derivative of PEG (DSPE-PEG) and tocopheryl polyethylene glycol 1000 succinate (TPGS) were employed. The mixed micelles were designed to act as a protective shield and increase drug delivery to tumor tissues. TPGS should improve the cell penetration capacity, and has been reported to increase the toxicity of docetaxel to cancer cells (24). TPGS may act synergistically to enhance the therapeutic efficacy of QUR loaded in the DSPE-PEG micelles. Small-sized, mixed micelles have the potential to effectively avoid reticuloendothelial system (RES)-based drug clearance (25).

In the present study, the objective was to improve the physicochemical and anticancer property of QUR against lung cancer cells. QUR was encapsulated in DSPE-PEG/TPGS-based mixed micelles and the physicochemical properties (size, shape and release kinetics) of the encapsulated QUR were examined. The anticancer activity of the drug-loaded micelles was evaluated in A549 lung cancer cells. The cytotoxic potentials of the free drug and drug-loaded micelles were compared using MTT assays and assessment of morphological cell density. The cytotoxic potential of the micelle formulation was further studied using a Hoechst 33342-based apoptosis assay.

\section{Materials and methods}

Materials. QUR was purchased from Sigma-Aldrich (Merck KGaA, Darmstadt, Germany). The DSPE-PEG component, 1,2-distearoyl-sn-glycero-3-phosphoethanolamine-N-methoxy (polyethylene glycol)-2000, was purchased from Corden Pharma (Cambridge, MA, USA). The specific TPGS used was D- $\alpha$-tocopheryl polyethylene glycol 1000 succinate from Eastman Chemical Co. (Kingsport, TN, USA). All other chemicals were reagent grade and used without further purification.

Preparation of QUR-loaded mixed micelles (QUR-M). The QUR-M was prepared by a solvent evaporation method. Briefly, QUR (10 mg), DSPE-PEG (90 mg) and TPGS (10 mg) were dissolved in $2 \mathrm{ml}$ chloroform and stirred for $10 \mathrm{~min}$. The organic mixture was rotary evaporated for $1 \mathrm{~h}$ leaving a thin polymer-lipid layer. The thin polymer-lipid layer was further vacuum evaporated for $1 \mathrm{~h}$. Following this, the resulting thin film was hydrated with phosphate-buffered saline (PBS; $\mathrm{pH}$ 7.4) and vortexed for $10 \mathrm{~min}$. The nanosuspension was immediately sonicated for $15 \mathrm{~min}$ at room temperature. The QUR-M were collected by centrifugation $(500 \mathrm{x} \mathrm{g} ; 10 \mathrm{~min}$ at $25^{\circ} \mathrm{C}$ ), washed with water twice and then lyophilized for use in further experiments. The unloaded drug in the supernatant was quantified by a HPLC method. HPLC analysis was performed using a Shimadzu-LC system (Shimadzu, Japan) equipped with an CBM-20A controller, LC-20AT pump, DGU-20A5 prominence degasser, SIL-20A auto sampler, SPD-20AV detector and CTO-10ASvp column oven at $25^{\circ} \mathrm{C}$. The column $(250 \mathrm{~mm} \times 4.6 \mathrm{~mm}, 5 \mu \mathrm{m})$ packed with silica and was protected with pre-column guard cartridge RP18 (30 y $4.6 \mathrm{~mm}, 10 \mu \mathrm{m}$; (PerkinElmer, Inc., Waltham, MA, USA). Acetonitrile and 2\% $\mathrm{v} / \mathrm{v}$ acetic acid $(\mathrm{pH} 2.60 ; 40: 60 \mathrm{v} / \mathrm{v})$ was used as a mobile phase and QUR was detected at $370 \mathrm{~nm}$. A $20 \mu 1$ sample was injected into the column at a flow rate of $1 \mathrm{ml} / \mathrm{min}$. The QUR-M were

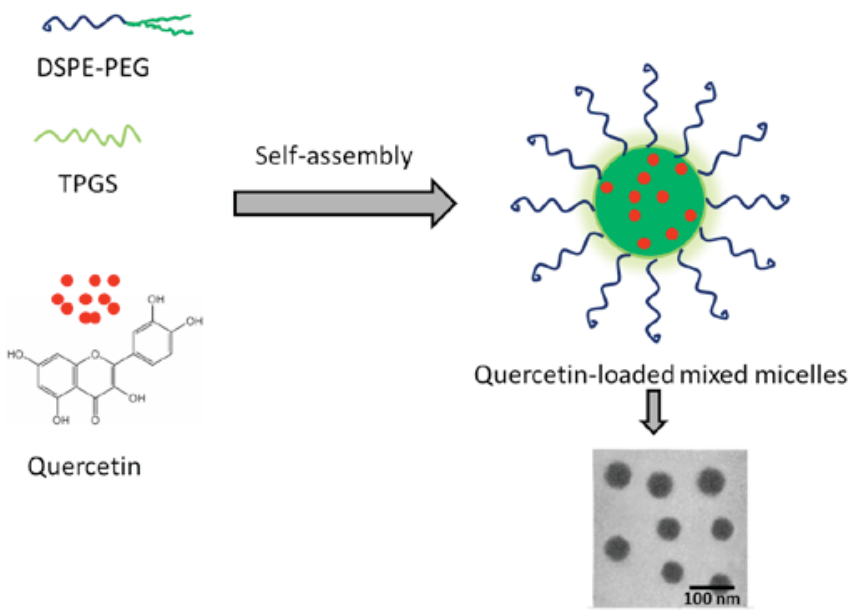

Figure 1. Graphical illustration of the preparation quercetin-loaded mixed micelles and transmission electron microscope image of the drug-loaded micelles. DSPE-PEG, 1,2-distearoyl-sn-glycero-3-phosphatidylethanolamine derivative of polyethylene glycol; TPGS, tocopheryl polyethylene glycol 1000 succinate.

found to have an active drug loading of $8.45 \%$ by weight with a loading efficiency of $>90 \%$.

Particle size analysis. The particle size and size distribution of the QUR-M were evaluated by dynamic light scattering (DLS) analysis using a Zetasizer Nano ZS system (Malvern Instruments, Ltd., Malvern, UK). The samples were suitably diluted (1:10) with water prior to experiments. The experiments were performed in triplicate.

Surface morphology analysis. The surface morphology of the QUR-M was investigated using a transmission electron microscope (TEM; JEM-2010; JEOL, Ltd., Japan). The TEM used an accelerating voltage of $120 \mathrm{kV}$. The samples were placed in a carbon-coated copper grid, counterstained with $2 \%$ phosphotungistic acid and air dried. Staining was performed at room temperature for $20 \mathrm{~min}$.

In vitro drug release assay. The in vitro drug release assay was performed using a dialysis method. In this assay, $2 \mathrm{mg}$ equivalent of QUR-M was resuspended in $30 \mathrm{ml}$ release medium (PBS, pH 7.4 and acetate-buffered saline, $\mathrm{pH}$ 5.0) and transferred to a dialysis tube. The sealed dialysis tube was placed in $30 \mathrm{ml}$ release buffer in a shaking water bath. The samples were withdrawn at specific time points $(1,2,4,8,12,24$ and $48 \mathrm{~h}$ ) and the amount of drug released was determined using HPLC, as described above. The percentage of QUR released was plotted against time.

Cytotoxicity assay. The cells were incubated in incubator maintained at $37^{\circ} \mathrm{C}$ and $5 \% \mathrm{CO}_{2}$ conditions. In the cytotoxicity assay, A549 cancer cells (ATCC, Manassas, VA, USA) were seeded in a 96-well plate at a seeding density of 10,000 cells/well and incubated for $24 \mathrm{~h}$. The following day, the cells were treated with blank micelles, free QUR and QUR-M in a concentration dependent manner and incubated for a further $24 \mathrm{~h}$. Untreated cells were observed as a positive control and DMSO-treated cells served as a negative control. 


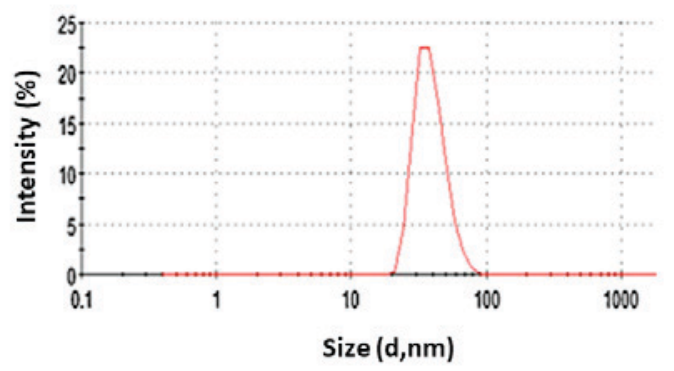

B

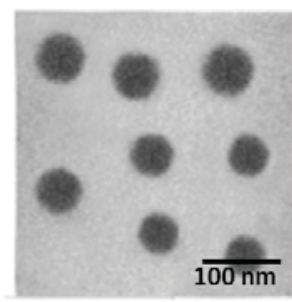

Figure 2. (A) Particle size distribution of quercetin-loaded mixed micelles. (B) The particle size distribution was determined by dynamic light scattering. d, diameter.

Following this, the medium was carefully suctioned off, $10 \mu 1$ 3-(4,5-dimethylthiazol-2-yl)-2,5-diphenyl-tetrazolium bromide (MTT; $5 \mathrm{mg} / \mathrm{ml}$ ) was added and the cells were incubated for $4 \mathrm{~h}$ at $37^{\circ} \mathrm{C}$. After this, $100 \mu 1 \mathrm{DMSO}$ was added to dissolve the insoluble formazan crystals that were produced by the action of mitochondrial succinate dehydrogenase. The absorbance of each individual well was quantified using a plate reader at $570 \mathrm{~nm}$. All experiments were performed in triplicate and results presented as the mean \pm standard deviation (SD). The IC50 value was calculated using GraphPad Prizm version 17 (GraphPad Software, Inc., La Jolla, CA, USA).

Apoptosis assay. The apoptosis assay was performed using Hoechst 33342-based nuclear staining and visualization of the cells using a confocal laser scanning microscope (CLSM). In this assay, $1 \times 10^{5} \mathrm{~A} 549$ cells/well were seeded in 12-well plate and allowed to attach for $24 \mathrm{~h}$. The following day, the cells were exposed to blank micelles, free QUR and QUR-M $(10 \mu \mathrm{g} / \mathrm{ml})$ and further incubated for $24 \mathrm{~h}$. The cells were washed twice with PBS and fixed with $4 \%$ paraformaldehyde. The cells were washed again prior to staining with Hoechst $33342(10 \mu \mathrm{g} / \mathrm{ml})$ for $10 \mathrm{~min}$. The nuclear morphology of the cells was visualized using a CLSM (TCS SP2; Leica Microsystems GmbH, Wetzlar, Germany).

Statistical analysis. Statistical analysis was performed using Student's t-test and one-way analysis of variance followed up by a post hoc Tukey's test. All results are expressed as the mean $\pm \mathrm{SD}$. $\mathrm{P}<0.05$ was considered to indicate a statistically significant result.

\section{Results and Discussion}

Characterization of QUR-M. The severe toxicity and insufficient therapeutic efficacy of chemotherapeutic drugs in cancer treatment necessitates the development of new therapeutic agents without toxic effects and with improved anticancer effects. Natural components from plant resources offer numerous options for the effective replacement of routine chemotherapeutic agents. QUR is a naturally occurring flavonoid that has exhibited potent antiproliferative effects on cancer cells without any effect on normal cells (16). QUR induces cancer cell apoptosis by blocking the cell cycle progression of cancer cells and modulating signaling

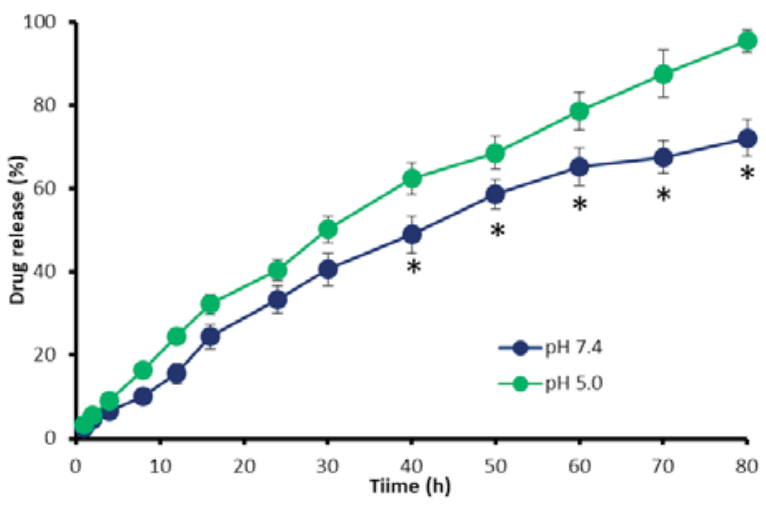

Figure 3. In vitro drug release profile of quercetin-loaded mixed micelles. The drug release assay was performed in phosphate-buffered saline and acetate-buffered saline at $37^{\circ} \mathrm{C} .{ }^{*} \mathrm{P}<0.05$ vs. pH 5.0.

pathways (20). However, the high hydrophobicity and short half-life span of QUR in the systemic circulation limit its therapeutic activity (17). Therefore, efforts are required to improve the physicochemical characteristics and anticancer effects of QUR. Thus, in the present study, QUR was encapsulated in DSPE-PEG/TPGS-based mixed micelles in order to improve its physicochemical and anticancer properties. PEG is likely to minimize the RES-based systemic clearance and increase the blood circulation time (26), while TPGS should improve the cell penetration capacity and increase the toxicity to cancer cells (27). TPGS may act synergistically with DSPE-PEG to increase the therapeutic efficacy of QUR loaded in the micelles (Fig. 1).

DLS analysis. The average particle size and particle size distribution were evaluated using DLS. The average particle size of the QUR-M was observed to be $70 \pm 1.5 \mathrm{~nm}$ with a polydispersity index of 0.142 , indicating that the particles were monodisperse (Fig. 2). A particle diameter of $<200 \mathrm{~nm}$ has been reported to be favorable for cancer-targeting applications (10). The QUR-M particle size of $<100 \mathrm{~nm}$ is likely to allow preferential accumulation in cancer tissues via an enhanced permeation and retention effect (15). The particle size will allow cellular uptake after intravenous administration (18). Moreover, the zeta potential was $-8.25 \pm 1.26 \mathrm{mV}$, which indicates that the QUR-M may avoid RES-based systemic clearance. 


\section{$24 \mathrm{~h}$}

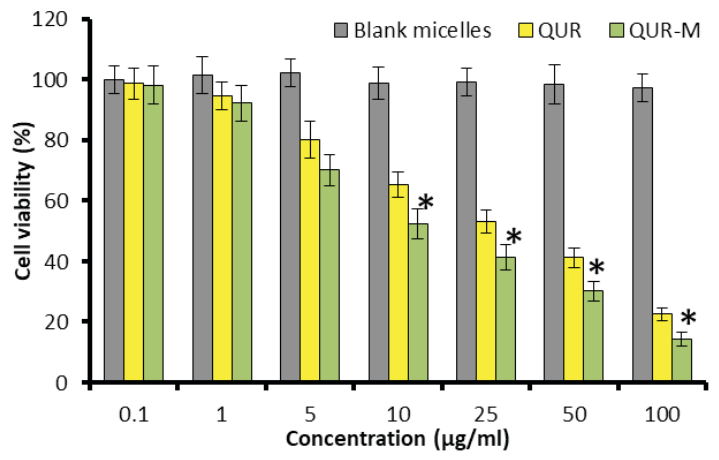

$48 \mathrm{~h}$

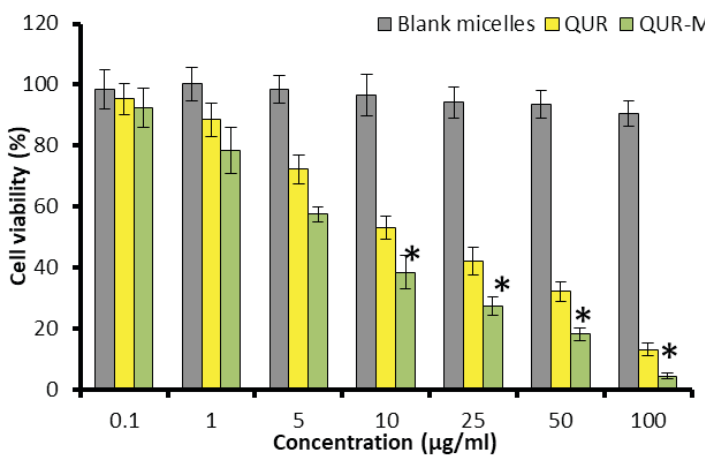

Figure 4. In vitro cytotoxicity of blank micelles, QUR and QUR-M in A549 cancer cells following incubation for 24 and 48 h. Data are expressed as the mean \pm standard deviation. QUR, quercetin; QUR-M, QUR-loaded mixed micelles. * $\mathrm{P}<0.05$ vs. QUR.

Control

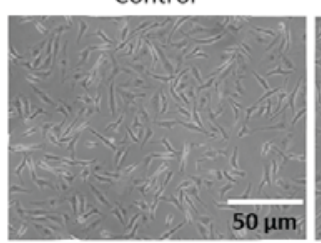

Blank micelles

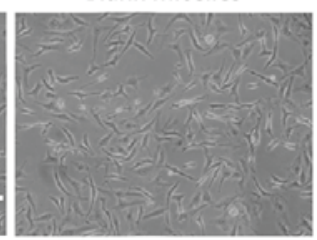

QUR

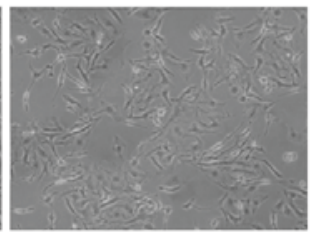

QUR-M

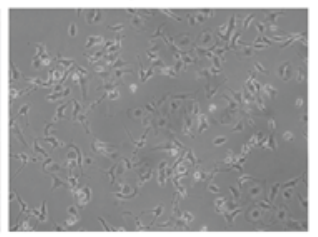

Figure 5. Morphological analysis of A549 cancer cells following treatment with blank micelles, QUR and QUR-M. QUR, quercetin; QUR-M, QUR-loaded mixed micelles.; Scale bar=50 $\mu \mathrm{m}$.
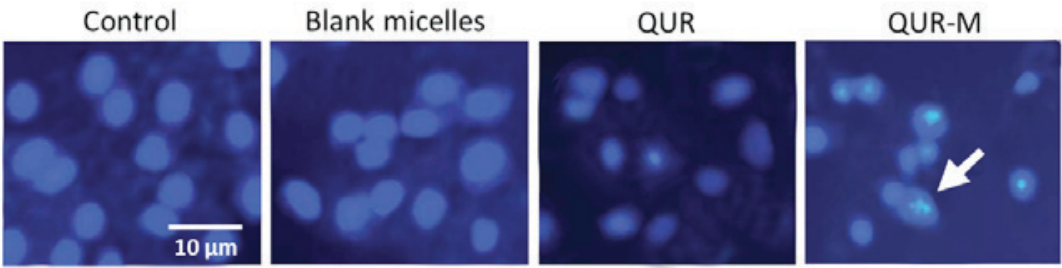

Figure 6. Nuclear apoptosis assay using confocal laser scan microscopy. The cells were treated with respective formulations and stained with Hoechst 33342 Scale bar $=10 \mu \mathrm{m}$.

Morphological analysis. The size and surface morphology of QUR-M were investigated by analysis using a TEM (Fig. 1). The particles were clearly spherical and appeared uniform in the copper grid. The particle size $(55 \mathrm{~nm})$ was slightly smaller when compared with that indicated by the DLS analysis, and the particles were observed to be monodisperse. The difference in particle size may be due to the folding of the PEG chain and aggregation of the micelles during the drying process.

In vitro drug release. The in vitro release of QUR from the QUR-M was investigated as pH 7.4 and 5.0 (Fig. 3). QUR was released in a controlled manner under the two $\mathrm{pH}$ conditions, indicating the stability of the mixed micelles. It was observed that $35-40 \%$ of the encapsulated QUR was released within the first $24 \mathrm{~h}$ of the release experiment. It may be noted that a slightly higher release rate was observed in acidic conditions. For example, $\sim 65 \%$ of the QUR was released in $\mathrm{pH} 7.4$ conditions after $80 \mathrm{~h}$ while $\sim 90 \%$ of QUR was released in $\mathrm{pH} 5.0$ conditions in the same time period. The increased release of QUR in acidic conditions is likely to be favorable to cancer-targeting applications (22). Overall, QUR-M exhibited a sustained-release profile, due to the stable incorporation of hydrophobic QUR in the core of the mixed micelles, which increased the path length. The sustained release of QUR may provide a stable concentration of the drug and prolong its therapeutic effects.

Anticancer effect of QUR-M in lung cancer cells. The anticancer effect of blank micelles and drug-loaded micelles in A549 lung cancer cells was evaluated by MTT assay. As shown in Fig. 4, free QUR and QUR-M exhibited a typical dose-dependent and time-dependent cytotoxic effect in A549 cancer cells. Notably, drug-loaded micelles exhibited a more potent anticancer effect $(\mathrm{P}<0.05)$ compared with that of free QUR at the two time points. The results demonstrated that the micellar system not only maintained the pharmacological action of QUR but also enhanced its anticancer effect. $\mathrm{IC}_{50}$ values were calculated to quantify the effects of the different formulations. The $\mathrm{IC}_{50}$ values of QUR and QUR-M were observed to be 12.45 and $6.42 \mu \mathrm{g} / \mathrm{ml}$, respectively. The superior 
cytotoxic effect of QUR-M may be attributed to the preferentially higher cellular uptake and controlled release of QUR in the intracellular environment. Following cellular internalization, QUR stored in the micelles may be gradually released, thereby exposing the cells to the drug for a prolonged time period. In addition, cells treated with blank micelles exhibited a high viability at all the concentrations tested, indicating the lack of toxicity and excellent biocompatibility profile of the micelles. A potent anticancer and time-dependent effect may be expected when a nanocarrier is internalized (28).

The cytotoxic effects of free QUR and QUR-M were further compared by morphological cellular imaging (Fig. 5). It was observed that control and blank micelle-treated cells were almost intact and maintained their typical morphology. The QUR-M induced a higher anticancer effect, compared with that of free QUR indicated by severe apoptotic body formation and a reduction in the number of cells present on the cover glass. Moreover, cells were rounded, which was indicative of cell death.

Apoptosis assay. The apoptosis potential of the formulation was tested using Hoechst 33342 staining of the lung cancer cells. The untreated and blank micelle-treated groups maintained a homogenous cellular morphology and no changes were observed (Fig. 6). The cells treated with free QUR or QUR-M, however, exhibited severe chromatin condensation and apoptotic body formation of the nuclei. The higher apoptosis potential of QUR-M may be attributed to its higher cellular uptake and the controlled release of the encapsulated QUR.

Conclusion. In summary, QUR-loaded mixed micelles were successfully prepared in order to improve the physicochemical properties and anticancer efficacy of QUR in lung cancer cells. The nanosized QUR-M exhibited $\mathrm{pH}$-responsive and controlled-release properties that may be beneficial in cancer treatment. The results of the present study clearly demonstrate that the QUR-M exhibited a superior anticancer effect compared with that of free QUR at 24 and $48 \mathrm{~h}$ time points in A549 cancer cells. The $\mathrm{IC}_{50}$ values of QUR and QUR-M were observed to be 12.45 and $6.42 \mu \mathrm{g} / \mathrm{ml}$, respectively. Consistent with this, cancer cells treated with QUR-M exhibited severe chromatin condensation and apoptotic body formation of the nuclei. Overall, the higher intracellular uptake, sustained drug release and presence of TPGS in the mixed micelles may inhibit cell proliferation and improve the therapeutic efficacy in lung cancers.

\section{Acknowledgements}

This study was funded by a research grant from the Third People's Hospital of Yancheng (Yancheng, China).

\section{References}

1. American Cancer Society. Cancer Facts \& Figures 2013. American Cancer Society, Atlanta, 2013. Available from: http://www.cancer.org/acs/groups/content/@epidemiologysurveilance/documents/document/acspc-036845.pdf.

2. Zarogoulidis K, Zarogoulidis P, Darwiche K, Boutsikou E, Machairiotis N, Tsakiridis K, Katsikogiannis N, Kougioumtzi I, Karapantzos I, Huang $\mathrm{H}$ and Spyratos D: Treatment of non-small cell lung cancer (NSCLC). J Thorac Dis 5 (Suppl 4): S389-S396, 2013
3. Miller KD, Siegel RL, Lin CC, Mariotto AB, Kramer JL, Rowland JH, Stein KD, Alteri R and Jemal A: Cancer treatment and survivorship statistics, 2016. CA Cancer J Clin 66: 271-289, 2016.

4. Vineis P and Wild CP: Global cancer patterns: Causes and prevention. Lancet 383: 549-557, 2014

5. Sridhar SS, Seymour L and Shepherd FA: Inhibitors of epidermal growth-factor receptors: A review of clinical research with a focus on non-small-cell lung cancer. Lancet Oncol 4: 397-406, 2003.

6. Grossi F, Gridelli C, Aita M and De Marinis F: Identifying an optimum treatment strategy for patients with advanced non-small cell lung cancer. Crit Rev Oncol Hematol 67: 16-26, 2008.

7. Otterson GA, Villalona-Calero MA, Hicks W, Pan X, Ellerton JA, Gettinger SN and Murren JR: Phase I/II study of inhaled doxorubicin combined with platinum-based therapy for advanced non-small cell lung cancer. Clin Cancer Res 16: 2466-2473, 2010.

8. Pilkington G, Boland A, Brown T, Oyee J, Bagust A and Dickson R: A systematic review of the clinical effectiveness of first-line chemotherapy for adult patients with locally advanced or metastatic non-small cell lung cancer. Thorax 70: 359-367, 2015.

9. Beecher GR, Warden BA and Merken H: Analysis of tea polyphenols. Proc Soc Exp Biol Med 220: 267-270, 1999.

10. Formica JV and Regelson W: Review of the biology of Quercetin and related bioflavonoids. Food Chem Toxicol 33: 1061-1080, 1995.

11. Hollman PC and Katan MB: Dietary flavonoids: Intake, health effects and bioavailability. Food Chem Toxicol 37: 937-942, 1999.

12. Gupta A, Birhman K, Raheja I, Sharma SK and Kar HK: Quercetin: A wonder bioflavonoid with therapeutic potential in disease management. Asian Pac J Trop Dis 6: 248-252, 2016.

13. Park MH and Min do S: Quercetin-induced downregulation of phospholipase D1 inhibits proliferation and invasion in U87 glioma cells. Biochem Biophys Res Commun 412: 710-715, 2011.

14. Zheng SY, Li Y, Jiang D, Zhao J and Ge JF: Anticancer effect and apoptosis induction by quercetin in the human lung cancer cell line A-549. Mol Med Rep 5: 822-826, 2012.

15. Anand David AV, Arulmoli R and Parasuraman S: Overviews of biological importance of quercetin: A bioactive flavonoid. Pharmacogn Rev 10: 84-89, 2016.

16. Lugli E, Ferraresi R, Roat E, Troiano L, Pinti M, Nasi M, Nemes E, Bertoncelli L, Gibellini L, Salomoni P, et al: Quercetin inhibits lymphocyte activation and proliferation without inducing apoptosis in peripheral mononuclear cells. Leuk Res 33: 140-150, 2009.

17. Gibellini L, Pinti M, Nasi M, Montagna JP, Biasi SD, Roat E, Bertoncelli E, Cooper EL and Cossarizza A: Quercetin and cancer chemoprevention. Evid Based Compl Altern Med 1011: 591356, 2011.

18. Puoci F, Morelli C, Cirillo G, Curcio M, Parisi OI, Maris P, Sisci D and Picci N: Anticancer activity of a quercetin-based polymer towards HeLa cancer cells. Anticancer Res 32: 2843-2847, 2012.

19. Tran TH, Ramasamy T, Truong DH, Shin BS, Choi HG, Yong CS and Kim JO: Development of vorinostat-loaded solid lipid nanoparticles to enhance pharmacokinetics and efficacy against multidrug-resistant cancer cells. Pharm Res 31: 1978-1988, 2014.

20. Ramasamy T, Choi JY, Cho HJ, Umadevi SK, Shin BS, Choi HG, Yong CS and Kim JO: Polypeptide-based Micelles for Delivery of Irinotecan: Physicochemical and in vivo characterization. Pharm Res 32: 1947-1956, 2015.

21. Ramasamy T, Ruttala HB, Gupta B, Poudel BK, Choi HG, Yong CS and Kim JO: Smart chemistry-based nanosized drug delivery systems for systemic applications: A comprehensive review. J Control Release 258: 226-253, 2017.

22. Choi JY, Ramasamy T, Kim SY, Kim J, Ku SK, Youn YS, Kim JR, Jeong JH, Choi HG, Yong CS and Kim JO: PEGylated lipid bilayer-supported mesoporous silica nanoparticle composite for synergistic co-delivery of axitinib and celastrol in multi-targeted cancer therapy. Acta Biomater 39: 94-105, 2016.

23. Ramasamy T, Kim J, Choi HG, Yong CS and Kim JO: Novel dual drug-loaded block ionomer complex micelles for enhancing the efficacy of chemotherapy treatments. J Biomed Nanotechnol 10: 1304-1312, 2014.

24. Tran TH, Ramasamy T, Choi JY, Nguyen HT, Pham TT, Jeong JH, Ku SK, Choi HG, Yong CS and Kim JO: Tumor-targeting, $\mathrm{pH}$-sensitive nanoparticles for docetaxel delivery to drug-resistant cancer cells. Int J Nanomedicine 10: 5249-5262, 2015. 
25. Zhou X, Luo S, Tang R, Wang R and Wang J: Diblock copolymers of polyethylene glycol and a polymethacrylamide with side-chains containing twin ortho ester rings: Synthesis, characterization, and evaluation as potential $\mathrm{pH}$-responsive micelles. Macromol Biosci 15: 385-394, 2015.

26. Corvazier E and Maclouf J: Interference of some flavonoids and non-steroidal anti-inflammatory drugs with oxidative metabolism of arachidonic acid by human platelets and neutrophils. Biochim Biophys Acta 835: 315-321, 1985.
27. Pan G, Lemmouchi Y, Akala EO and Bakare O: Studies on PEGylated and drug-loaded PAMAM dendrimers. J Bioact Compat Polym 20: 113-128, 2005.

28. Yamazaki M and Ito T: Deformation and instability in membrane structure of phospholipid vesicles caused by osmophobic association: Mechanical stress model for the mechanism of poly(ethylene glycol)-induced membrane fusion. Biochemistry 29: 1309-1314, 1990. 